[To be pubished in: The Electronic Library. Vol 33. Issue 4. 2005]

[This article is (c) Emerald Group Publishing and permission has been granted for this version to appear in http://openaccess.uoc.edu. Emerald does not grant permission for this article to be further copied/distributed or hosted elsewhere without the express permission from Emerald Group Publishing Limited.]

\title{
Wikipedia at University: what faculty think and do about it
}

\author{
Eduard Aibar, Josep Lladós, Julià Minguillon, Antoni Meseguer and, Maura Lerga \\ Universitat Oberta de Catalunya
}

\begin{abstract}
Structured Abstract:
\end{abstract}
Purpose - The purpose of this paper is to investigate university faculty perceptions and practices of using Wikipedia as a teaching resource.

Design/methodologylapproach - This study is based on a large survey to all faculty members in two large public universities. A total of 913 valid responses were collected through an online questionnaire with 9 control variables and 41 Likert-scaled questions.

Findings - Our results do not support an overwhelming sceptical attitude among faculty towards Wikipedia. The overall quality of Wikipedia articles is highly valued and most faculty are regular users, just as students are. Though most faculty show a positive view on the teaching usefulness of Wikipedia, few of them actually use it for teaching purposes. We have detected a certain conflict between standard academic procedures of knowledge building and the open collaborative model on which Wikipedia rests. In the end, two important factors play a role in shaping faculty views: (a) their colleagues' perceived opinions and practices and (b) academic disciplines.

Research limitations/implications - The survey has only been conducted in two universities. More institutions are needed in order to broaden the scope.

Originality/value - We have gathered a greater number of answers than those collected in previous studies. The questionnaire is also very extensive. The survey has been addressed to all faculty members at one online university and at one standard brick-and-mortar university.

Keywords: Higher education, Academic Staff, Encyclopaedias, Teaching, Open Educational Resources, Peer Production

Article Classification: Research paper 


\section{Introduction}

A key impact the Internet has had on university education is the vast availability of open educational contents - course materials, study guides, collections of exercises, etc. - accessible on the network and free for everyone. This phenomenon began in 2001 with the creation of the initiative OpenCourseWare (OCW) at MIT (Massachusetts Institute of Technology) and has evolved into the socalled Open Educational Resources movement.

MIT's initiative has spurred many universities everywhere to start similar projects and thus contribute to the international movement of open educational resources. The emergence of Web 2.0 has also opened up a wide range of new possibilities that may end up influencing decisively in learning processes, both inside and outside formal academic institutions. Among other impacts, Web 2.0 initiatives have blurred the traditional boundary between producers and consumers of information and knowledge.

Wikipedia precisely represents the junction where these two trends converge. From one perspective it can be depicted as a gigantic open repository of knowledge and information - with great potential for use in learning processes at all levels of education (Saorín Pérez et al. 2011, Konieczny 2014) - and from another it has become a prime example of collective construction of knowledge, through a virtual platform that facilitates collaboration on an unprecedented scale. In fact, Wikipedia can be taken as a canonical example of what Benkler (2006) has called mass-online commons-based peer production (or, in the short form, peer production): a particular mode of producing information and knowledge goods that has its origins in free software projects.

Peer production can be characterized as a form of voluntary cooperation aimed at building some kind of common resource, through the intensive use of networking platforms and tools. In contrast to traditional institutions, peer production initiatives work by means of non-hierarchical and highly decentralized organizations. They are also based on a particular understanding of copyright as a right for distribution (Weber, 2004), not as a right to exclude. Entry barriers to participation are usually low and formal accreditations are mostly irrelevant. Authority and influence in peer production projects come mainly from the quality of individuals' past contributions - as judged by other peers.

The great success of some peer production projects - GNU/Linux or Wikipedia - has led the application of this model to other realms of human activity (including hardware building and design). A number of recent initiatives are trying to import, sometimes explicitly, peer production mechanisms to the sphere of science: from citizen-science projects, to the movements for open research and open access (Nielsen, 2011). Similarities between science and peer production are clear: both fields rely heavily on the open publication of results, on peer review as an essential mechanism for quality control, and have been described as meritocratic cultures. Yet there are also important differences between them; among others, authorship in peer production is somehow diluted as a result of massive collaboration and anonymous contributions, and peer review is mostly done post-publication in an open (not blind) way.

In order to explore the compatibility and possible tensions between science and peer production we have translated the problem into a more manageable research issue: analysing the way an important part of science - university faculty members - perceive and use for teaching purposes a particular instance of peer production; namely, Wikipedia.

\section{Wikipedia in higher education}

Wikipedia is currently the most important website for general consultation and is contributing positively to learning processes, both inside and outside academia. In fact, since the Internet is now the general public's most important information source for science and technology issues (Brossard and Scheufele, 2013), and since most search engines place Wikipedia entries in their top results, it is highly likely that the free encyclopaedia has also become the most important channel for the public's understanding of science.

In fact, in the university context it is one of the most employed resources by students who use it regularly as a reference tool to carry out different assignments and tasks (Brox, 2012; Knight and 
Pryke, 2012). This is not only due to the quality of many of its articles, but also to the easy access to its content, the hypertext structure that facilitates navigation and the abundance of references and sources, according to Alonso and García (2013) and Lim (2009). Based on a representative online survey among 4,400 students from German universities (return rate 40\%), Wannemacher and Schulenburg (2010) found that $80 \%$ of students use Wikipedia on a regular basis and $60 \%$ use it frequently or very frequently.

But despite students' broad and intensive use, the attitude of university faculty does not seem so positive. There is widespread opinion that academics perceive Wikipedia with some scepticism. It is known that many academics believe, for example, it is illegitimate to cite Wikipedia as a source, because their articles do not have clear and identifiable authorship, and so it is difficult to verify their content (Jaschick, 2007). Others do not like their students to use it and even try to ban it in their courses. Unfortunately, empirical studies on faculty perceptions and uses of Wikipedia in learning environments are few and limited in scope.

Based on a survey of 14 university instructors, An and Williams (2010) identified both educational benefits and major barriers of using 2.0 tools. Some benefits mentioned are: fostering of interaction; communication and collaboration among students; improvement in writing and technological skills; the ease of use and flexibility; and a new role for teachers as facilitators of learning rather than distributors of knowledge. The major barriers are a perceived unease with openness among some students, the lack of institutional technical support for faculty and the time needed to learn and manage new tools.

Dooley (2010) notes that faculty negative attitude towards Wikipedia is usually based on a perception of inaccuracy of its content and also on its potential for discouraging students from using other more reliable sources of information. Her survey - with 105 respondents - shows that only $7 \%$ use Wikipedia frequently for teaching or research tasks. In a similar vein, H. Chen (2010) identifies credibility as university faculty's main concern about Wikipedia and highlights academic disciplines as a key factor in explaining attitudes towards Wikipedia. This study also shows that age correlates with more negatives views and that faculty who frequently use other online resources are more sceptical about Wikipedia.

In a qualitative study based on five interviews, Bayliss (2013) proposes two main causes of the cautionary and cynical attitude towards Wikipedia. First: the lack of knowledge and poor understanding of Wikipedia editing processes and policies by academics; and second, a negative attitude toward collaborative knowledge production occurring outside academia. Along the same lines, Knight and Pryke (2012), following a survey of 133 faculty members, state that the main reasons for academics to distrust Wikipedia is its obvious departure from "conventional models of scholarship". They mention the no-need of accreditation for contributors, the possibility of anonymous editing, the absence of formal pre-publication peer review, and the blurred authorship of entries. Most faculty members tend to favour a 'low-stakes' use by students (like the initial scoping of an issue), over higher-stakes (such as citing facts or as a serious source of knowledge and references).

Other possible explanations of the negative attitude towards Wikipedia have to do with its particular way to produce and assess knowledge content, something that is closely linked to the peer production model it represents. Beyond specific accuracy and credibility concerns, a more fundamental conflict on epistemological and power grounds is detected by several authors (Black, 2008; S. Chen, 2010; Eijkman 2010). Based on a survey with 99 respondents, Eijkman (2010) shows that a majority of academics display "a blend of relatively cautious acceptance and/or gentle discouragement" towards Wikipedia. Surprisingly, the study finds a slight negative correlation between knowledge of Wikipedia and favourable views of it, and that 'soft-science' academics - allegedly more prone to a social constructivist view of knowledge - show a more negative attitude than their 'hard-science' fellows. However, his main point is that, for faculty members, Wikipedia has become a symbol of opposition to the traditional power-knowledge arrangements in academia.

\section{Aims and research design}

We are undertaking a research project in order to systematically analyze, using a comprehensive empirical study, the perception and attitudes of university faculty from different scholarly areas towards Wikipedia. The study aims to investigate relationships between these perceptions and several faculty 
characteristics in order to establish the extent to which the sceptical attitudes are related to: (a) personal or generational factors or (b) an implicit conflict between the standard scientific or academic epistemological stands and the specific peer-to-peer culture of Wikipedia (as a paradigmatic example of content production in a collaborative open network).

We focus our attention on faculty members. In particular, we want to know if they perceive Wikipedia as useful for teaching, if they actually use it for teaching purposes and what they think about students using it. Other related issues we want to address are what faculty members think about the editing system, how they assess Wikipedia's overall quality and whether they think Wikipedia is compatible with academic standards.

We also want to explore faculty's attitude towards open collaboration and other web 2.0 tools and identify possible incentives that would be appropriate for fostering the teaching uses of Wikipedia. Finally, we have searched for possible associations between actual teaching use of Wikipedia and (a) a number of perception and usage variables and (b) some personal and professional characteristics of faculty members.

As the central part of this study, we have conducted an online survey to all faculty members of the Universitat Oberta de Catalunya (UOC) and the Universitat Pompeu Fabra (UPF). UOC, launched in 1994, is a purely virtual public university providing higher and continuous education to more than 60,000 students by means of a hierarchical structure composed of (approximately) 250 full-time professors and almost 2,000 part-time associate professors. Some of these associate professors also teach in other brick-and-mortar universities but all of them are considered as faculty members. UPF was launched in 1990 and it is the most recent public university created in Catalonia. It has around 12,000 students and there are 1,511 faculty members.

As shown in Table 1, we had 913 valid responses from a universe of 3,639 individuals. To our knowledge, this is the largest number of responses ever gathered from university faculty members about Wikipedia. For a confidence level of $95 \%$, and the assumption of maximum uncertainty $(p=q=$ $0.5)$, the margin of error is $2.81 \%$.

Table 1. Technical information on the questionnaire

\begin{tabular}{|c|c|}
\hline Study universe & $\begin{array}{l}\text { Faculty members of the Open University of } \\
\text { Catalonia (UOC) and Universitat Pompeu } \\
\text { Fabra (UPF) }\end{array}$ \\
\hline Study universe size & 3,639 \\
\hline Method & $\begin{array}{l}\text { Online survey sent to the universe, with no } \\
\text { quota groups }\end{array}$ \\
\hline Sample size & 913 \\
\hline Sampling error & $\begin{array}{l} \pm 2.81 \% \text { for overall data in the case of } \\
\text { maximum uncertainty }(p=q=0.5) \text {. Confidence } \\
\text { level } 95 \% \text {. }\end{array}$ \\
\hline Resulting sample & Not weighted \\
\hline Date of launching & $\begin{array}{l}\text { November 19, } 2012 \text { (UOC) and April 29, } 2013 \\
\text { (UPF) }\end{array}$ \\
\hline Data collection & $\begin{array}{l}\text { From November } 19 \text { to December } 3,2012 \\
\text { (UOC) and from April 29, } 2013 \text { to May 16, } \\
2013 \text { (UPF) }\end{array}$ \\
\hline
\end{tabular}


The questionnaire was organized in two parts. The first part collected data on gender, age, area of expertise, $\mathrm{PhD}$ degree, years of experience in university teaching and academic position. The second part, with 41 questions, was aimed at gathering information about perceptions, practices and other aspects that could affect the teaching use of Wikipedia in higher education. These questions had to be answered via a 5-point Likert scale. Depending on the nature of the questions, this scale referred to the level of agreement or disagreement with a statement (1="Strongly disagree" and $5=$ "Strongly agree") or to the frequency of certain actions (1="Never" and $5="$ Very often").

In order to design the final version of the questionnaire, an exploratory qualitative study was carried out involving twelve interviews to faculty members - selecting two from each of the six main schools at UOC. Comments and suggestions collected through this procedure helped to improve the survey until it reached its final form. These interviews were conducted between October 12 and 16, 2012. The online survey was launched on November 19 and remained open until December 3, 2012, for UOC faculty, and from April 29 until May 16, 2013, for UPF faculty.

As for the methods used in this study we have mainly developed a descriptive statistical analysis in order to provide basic information about the main characteristics of the sample. We have also used a correlation analysis to demonstrate the existence of associations between some variables that identify faculty perceptions and uses. In particular, we have chosen the polychoric correlation as a measure of interdependence between two different variables.

In order to investigate the influence of personal variables on the decision to use Wikipedia for teaching, we have also developed an analysis of variance (ANOVA) for testing the statistical significance of differences in the mean value among different categories of individuals.

\section{Main findings}

In this section we present the most important results of our descriptive analysis - grouped by the theoretical constructs used in the study - as well as some associations between variables that seem particularly relevant.

\subsection{Wikipedia as a teaching tool}

Three questions in the survey were designed to measure the perceived usefulness of Wikipedia as a teaching tool. $46.8 \%$ of respondents agree [1] that Wikipedia is useful for teaching, while only $18.8 \%$ disagree $(N=908)$. When asked for particular benefits the answers are a bit less positive: $35.9 \%$ agree it fosters new skills for students, while $27.7 \%$ disagree, and $36.0 \%$ think it improves students' learning while $26.4 \%$ do not think so $(N=902)$.

There is a sharp contrast between the perception of teaching utility - rather positive - and the actual teaching practices of faculty members, which is, as expected, very low. A vast majority of faculty has never or seldom used Wikipedia for teaching assignments (75.7\%). Only about $9.0 \%$ say they use it frequently or very frequently for that purpose $(N=896)$. Wikipedia is used a little bit more as an information source for developing teaching materials: the percentage of faculty who never or seldom use it is about $66.9 \%$ while frequent users rise to $11.9 \%(N=899)$. 
Figure 1. Wikipedia in teaching

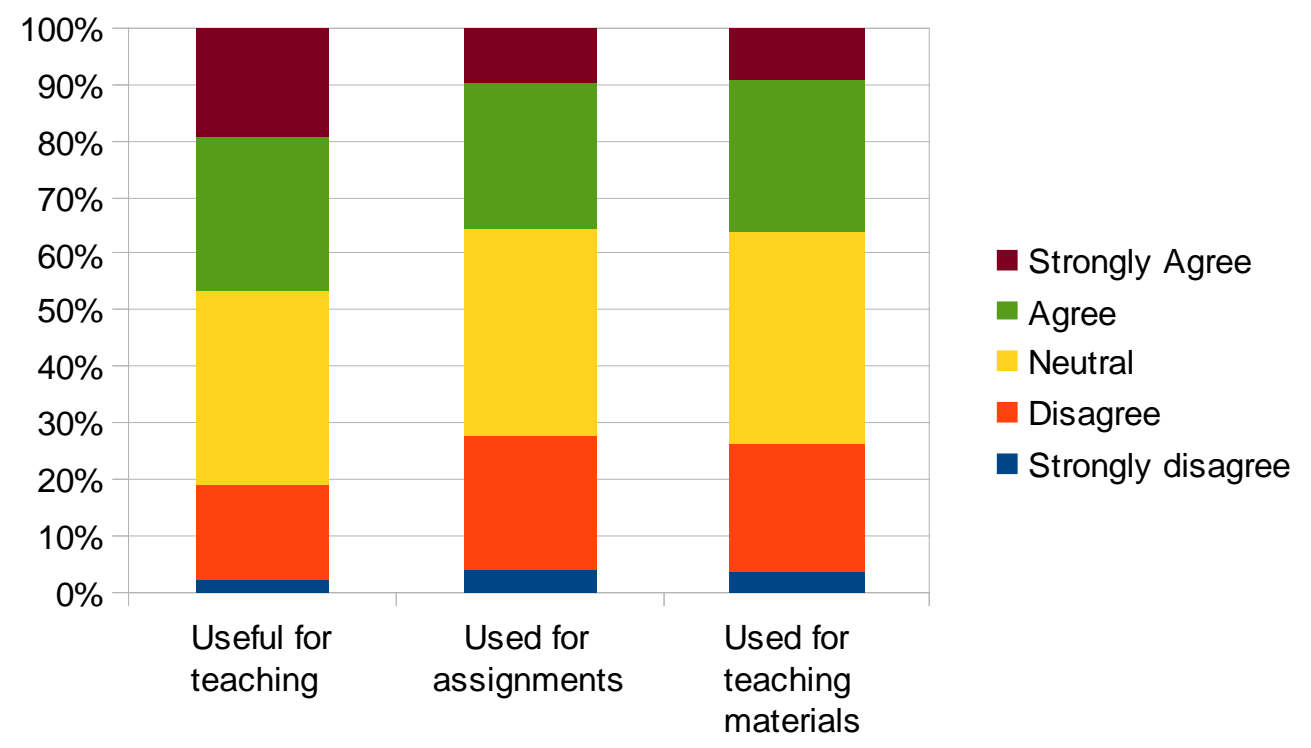

\subsection{Recommending and citing Wikipedia}

Since previous literature has shown that some faculty explicitly discourage the use of Wikipedia among students and also that some even ban citing it in essays and assignments, we have included in our survey some questions about both issues - recommendation and citing. When asked about their willingness to recommend to students to consult Wikipedia, almost half of the respondents (46.5\%) never or seldom do, while about $27.2 \%$ do it frequently or very frequently $(N=904)$. They are even less eager to recommend Wikipedia to their own colleagues - only $23.5 \%$ do it frequently $(N=890)$. Nevertheless, most of them (46.0\%) do not see any problem in students using Wikipedia and $23.1 \%$ do not feel comfortable about it $(N=898)$.

This last result contradicts the common idea that most faculty are completely against students consulting Wikipedia or that many of them eventually ask them not to use it. Although $23.1 \%$ do not like students to do so, only $13.7 \%$ completely disagree with them using it.

Nevertheless, when asked about citing Wikipedia in their academic output, faculty show a clear negative position. The biggest percentages) are for those that never do it (44.9\%) and those who seldom do it (24.5\%). Only a handful do it very frequently (4.1\%) or frequently (8.7\%). It is worth noting that when phrasing the question we did not specifically ask about research papers, but about academic texts in general - which includes teaching materials and less formal pieces of work too) $(N=905)$.

\subsection{Ease of use and editing system}

We have also explored the perceived ease of use of Wikipedia. As expected a vast majority of faculty members think Wikipedia is easy or very easy to use (87.3\%) and most of them also think it is rather straightforward to find the information they are looking for $(N=909)$. But while this passive use for consulting shows no usability problem, active use involving editing - providing new or modifying existing content - is perceived rather differently. About $14.0 \%$ disagree that editing Wikipedia is easy while $42.5 \%$ agree $(N=816)$. Since, as we will see later, few of them actually contribute to Wikipedia 
and we may assume the number of faculty who have ever tried to edit it is not much higher, we have to view) these figures with care.

There was also a question about the editing and reviewing system in Wikipedia. Here $31.8 \%$ of the respondents say they trust the system while a slightly lower percentage, $26.8 \%$, declare they do not $(N=884)$. The greatest number is for those who are undecided $(41.4 \%)$. It is worth noting that the $6.1 \%$ who strongly disagree (about trusting the system), outnumber the $5.3 \%$ who strongly agree. However since the percentage of respondents who choose the midpoint answer is substantial, we can hypothesize that most faculty do not actually know Wikipedia's specific editing system very well nor the way the peer-review process works. This could explain their lack of a clear opinion and is also consistent both with the results of the qualitative interviews in our exploratory study and with the low percentage of faculty members editing (and thus contributing) to the content in Wikipedia.

\subsection{Quality assessment}

Since previous literature has remarked that most faculty show serious concerns about Wikipedia's credibility, accuracy and reliability, four questions in the survey were designed to measure how the quality of Wikipedia is perceived by academics. Three of the questions were specifically addressed to the quality assessment of articles - we did not ask for particular articles or entries, but for their opinion on the overall encyclopaedia. We first asked about the articles' reliability: $38.4 \%$ of respondents agree that information contained in Wikipedia articles is reliable, $20.6 \%$ do not agree and $41.0 \%$ are of an opinion somewhere between the two; thus, the answers are leaning towards a fairly positive view $(N=906)$. Second, we asked if information deployed in the articles was updated; the answers we received were substantially more positive: $48.4 \%$ think it is updated, only $13.8 \%$ think it is not and $37.8 \%$ choose the midpoint $(N=903)$. Third, there was a question asking about how comprehensive the articles are: this was the feature most negatively assessed and the only one where affirmative answers were outnumbered by negative ones. Specifically, $27.3 \%$ agree or strongly agree that articles are comprehensive, $29.4 \%$ disagree or strongly disagree and the rest $-43.3 \%$ - take the midpoint $(N=898)$.

Figure 2. Quality assessment

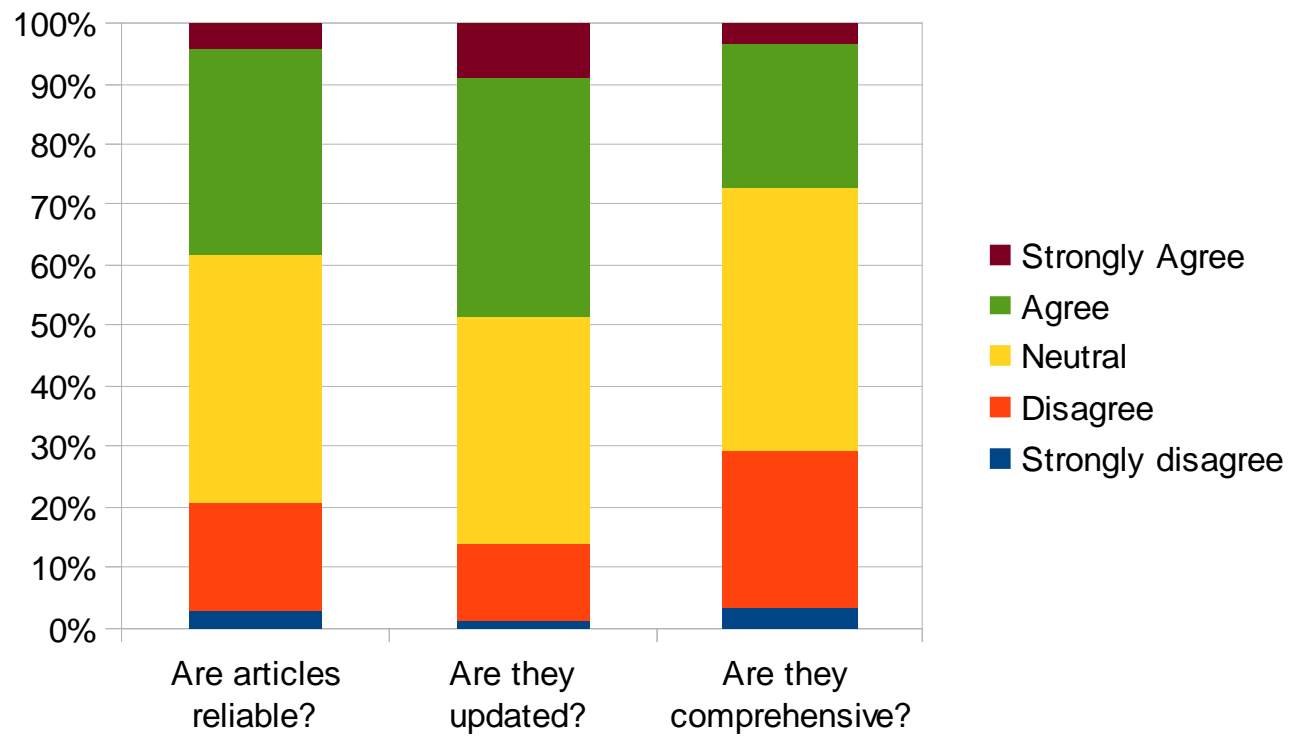


These results altogether do not at all support the idea - highlighted by previous studies and commonly believed - that university faculty are really concerned about quality standards in Wikipedia and often take a sceptical or even plainly negative attitude. Most faculty actually think the overall quality of Wikipedia articles is relatively respectable. When quality assessment is decomposed in more specific factors, results are very apparent. Though articles, in general, are deemed a bit more uncomprehensive, they are clearly considered reliable and updated. A strong negative perception of quality is only held by a minority.

\subsection{Active and passive use}

Another set of questions in the survey was devoted to explore the previous user experience with Wikipedia. We wanted to explore both active (editing and contributing) and passive uses of Wikipedia (mainly searching and browsing).

Passive use was split into three questions: consulting Wikipedia (1) for topics related to their own field of expertise, (2) for other academic fields and (3) for personal matters. Here a decreasing pattern is found that diminishes from personal matters (the most consulted area) - to their own field of expertise (the least consulted). In personal matters more than $62.6 \%$ of respondents declare they visit Wikipedia frequently or very frequently while less than a mere $18.1 \%$ say they never or seldom visit it $(N=900)$. A majority of faculty (55.3\%) also use Wikipedia to search for information about other academic matters not in their field of expertise, whereas $20.1 \%$ never or rarely do it $(N=902)$. When asked about consulting Wikipedia for issues related to their own academic discipline, only $38.1 \%$ do it frequently or very frequently while a slightly lower percentage, $37.1 \%$, say they never or seldom do $(N=900)$.

These data on the frequency of visits to Wikipedia are certainly striking. On the one hand, they clearly show that most faculty are regular users of the encyclopedia and thus deny, or at least call into question, the mostly negative or plainly skeptical attitude commonly attributed to them. On the other hand, the figures on the frequency of consultation are virtually the same as those provided by the largest studies undertaken on university students. Wannemacher and Schulenburg (2010), in their large survey, report that $60 \%$ of the students use Wikipedia frequently or very frequently. There seems to be no difference between faculty and students in their respective use of the free encyclopedia.

The data also confirm our initial expectation that faculty mainly uses Wikipedia for consulting matters not strictly belonging to their field of expertise - where they are more acquainted with the basic facts and concepts usually provided by encyclopaedic entries and also have other more specific sources of information (academic and scientific literature, mainly). They use Wikipedia, in their professional activities, primarily for quick introductory and basic information in areas beyond their own disciplines.

Active use (contributing to Wikipedia), is scarce, as expected. In fact, from all frequency questions in the survey, it shows the biggest rate of 'never' answers: $64.5 \%$. Taken together, the 'never' and 'seldom' answers amount to $84.1 \%$ while only $5.8 \%$ of respondents say they frequently contribute to Wikipedia $(N=899)$. Very frequent editors amount to just $1.7 \%$, a figure that closely resembles the one found for students in the above-mentioned survey (1\%). 
Figure 3. Passive and active use

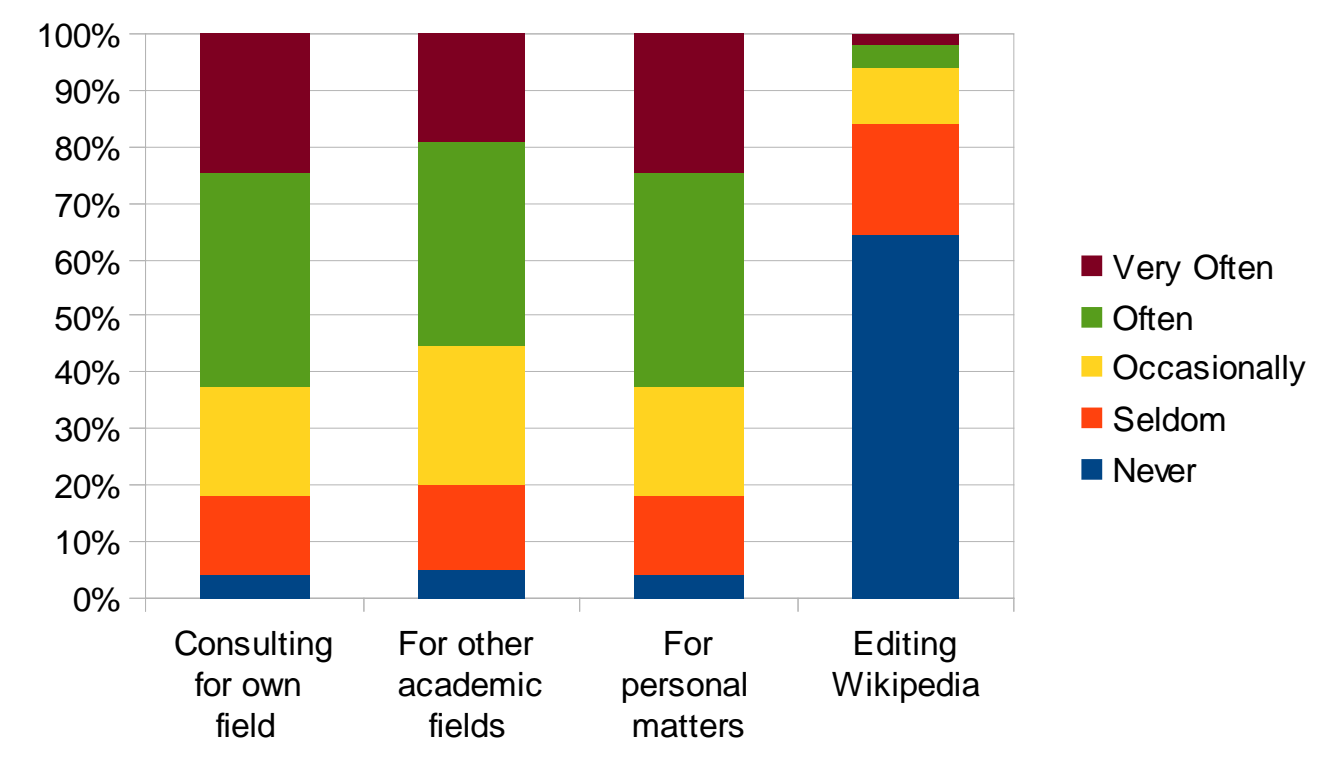

\subsection{The social image of Wikipedia within academia}

An important set of questions addressed the issue of the social image of Wikipedia within academic culture. First, a clear majority, 53.2\%, of faculty members think the use of Wikipedia is not well considered by their colleagues, while only $13.8 \%$ think it is $(N=891)$. We also explored faculty perception of their colleagues' actual use of Wikipedia, in order to contrast it with the previous item. Though most respondents $(N=856)$ do not take a clear side $(40.0 \%$ choosing the midpoint), those that think colleagues do not use it much (34.0\%) significantly outnumber those who think they do (26.0\%). 
Figure 4. Image within academia

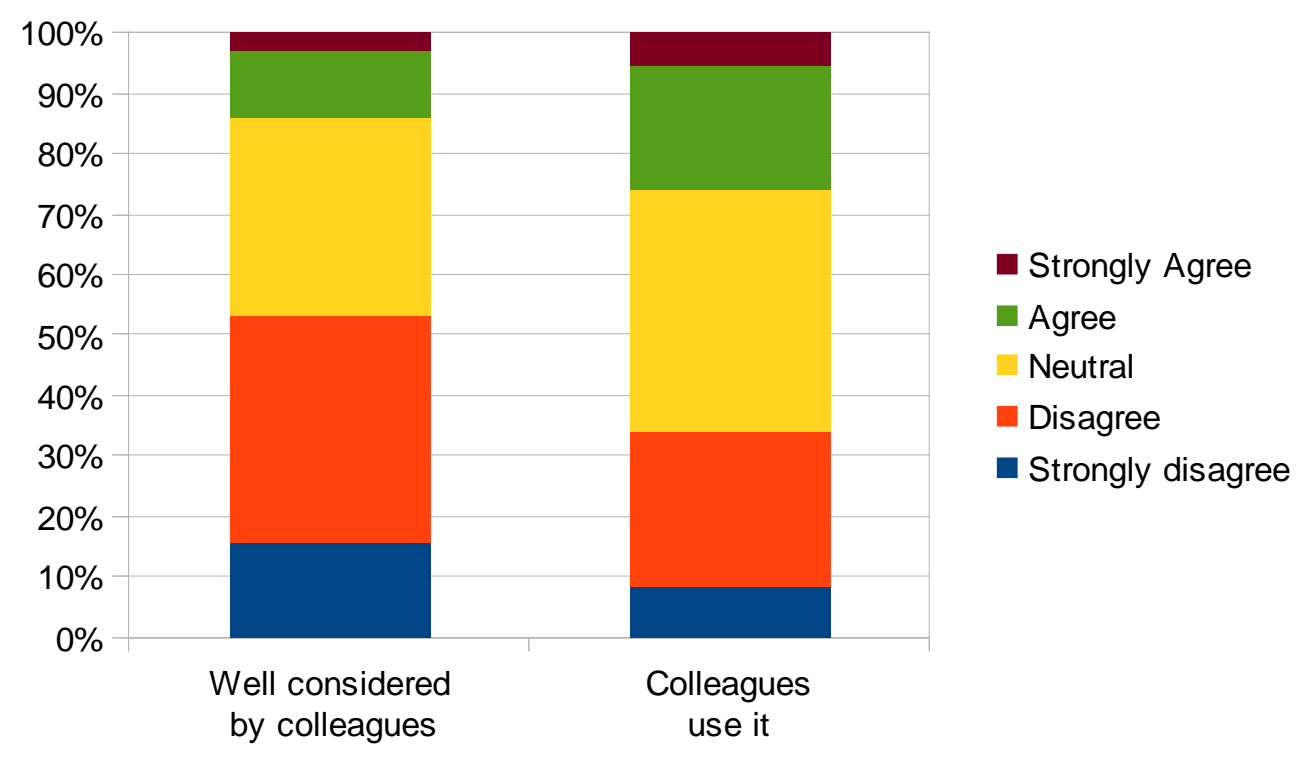

This is indeed in sharp contrast with our own data on actual use: though few faculty think their colleagues are really using Wikipedia, we notice that most of them do. It is clear that the majority perception that Wikipedia is not well considered by their colleagues prevents many academics to publicly acknowledge its use and, even less, to recommend it. As we mentioned before, only a few actually recommend Wikipedia to their students $(27.2 \%)$ and fewer $(23.1 \%)$ recommend it to their own colleagues.

\subsection{Sharing, collaboration and web 2.0 tools}

We have also included three more questions in the survey regarding what we call "sharing and collaborative attitude". First, we asked faculty to rate the importance of openly sharing academic content on the web $(N=902)$. A vast majority agree this is important $(84.8 \%)$ while only a few think it is not (3.4\%). We also inquired if they think it is also important to publish research results in other media and not only in academic journals $(N=901)$. A similar, though a bit smaller, clear majority also gave positive answers (77.5\%). Finally, we added a third question into this section to find out how important they consider it to be for students to become familiar with collaborative environments on the Internet. From these three items this was the one that got the greatest agreement: $86.6 \%$ agree or strongly agree this is important $(N=902)$.

Nevertheless, when asked if the open sharing of teaching resources is welcome in academic culture, though answers are still apparently positive, the difference with the negative position is not so sharp: $45.4 \%$ agree and $24.1 \%$ do not $(N=893)$. Again, it seems that the use of Wikipedia is seen as less socially acceptable within academic culture, when compared to other open educational resources.

Three further questions were included in order to evaluate the degree of use of web 2.0 tools. First, we asked about contributing to blogs $(N=902)$. Only $19.4 \%$ of faculty declare they contribute frequently to blogs and a broad majority never do it (37.7\%) or seldom do it (24.2\%). A similar negative answer was obtained when asked about publishing academic content in open environments: $28.6 \%$ never do it and $22.1 \%$ only do it seldomly, while $8.7 \%$ do it very frequently and $17.0 \%$ frequently $(N=899)$. The third question inquired about active participation in social networks. Here, the percentage of non-users and low users was significantly smaller ( $18.4 \%$ and $24.6 \%$ respectively), while frequent users were slightly higher $(33.3 \%)$ than in previous items $(N=907)$. 


\subsection{Institutional context and possible incentives}

A last section in the survey was devoted to the topic of institutional support and possible incentives to use open environments in teaching and, specifically, Wikipedia as a teaching tool. Institutional support does not seem to be a real concern, since more than $62.4 \%$ of faculty think their own university promotes the use of such open environments. Only $14.7 \%$ does not agree with the statement $(N=886)$. We also inquired about the extent to which their university considers the use of those environments as a teaching merit. Answers on this issue were clearly less positive (agreement was declared by $38.6 \%$ and disagreement by $29.2 \%)$, though they still outnumber the negative view $(N=860)$.

Possible incentives were also explored by asking the extent to which four specific factors would help them to design educational activities using Wikipedia. The four factors were: (1) having a catalog of best practices, (2) having colleagues explaining their own experiences, (3) receiving specific training, and (4) getting more institutional recognition. The factor that got the best assessment was "having a catalog presenting best practices" where $63.8 \%$ agree or strongly agree with the statement $(N=878)$. The second best assessment was for "getting greater institutional recognition" with $52.0 \%$ showing their agreement $(N=871)$. Experience explanations by colleagues was third with a very similar distribution of answers - agreement by $51.3 \%(N=878)$ - and the least valued was specific training, although a majority of faculty (49.9\%) still declared their agreement $(N=876)$.

\subsection{Factors associated with using Wikipedia as a teaching tool}

As mentioned in the methods section we have also explored possible associations between variables in our study. In particular we have developed correlation tests to check associations between the teaching uses of Wikipedia - the latent variable encompassing consulting Wikipedia to develop teaching materials, and planning assignments involving Wikipedia - and a number of other variables: (1) quality perception - encompassing reliability, updating and comprehensiveness of articles-, (2) perception by colleagues, (3) use by colleagues, (4) improving students learning, (5) editing Wikipedia, (6) consulting Wikipedia for personal matters and (7) feeling comfortable about students using it for their tasks. We have also checked correlations between quality perception and the rest of the variables.

First of all, the teaching use of Wikipedia is associated with its perceived quality, though the correlation is only moderate $(r=0.40, N=874, p<0.001[2])$ and certainly lower than we expected. Since quality concerns among faculty have been repeatedly highlighted by previous literature, we thought the small percentage of faculty who actually uses Wikipedia as a teaching tool were also going to make a more positive assessment of its overall quality. They do, but not to a great extent - quality perception does not seem to be so influential and other factors might be more relevant.

The strongest correlation for the teaching uses is, nevertheless, the active use of Wikipedia, i.e. making editions or contributions $(r=0.59, N=887)$. Passive use (consulting) is only weakly associated $(r=0.30, N=886)$. 
Table 2: Correlations between teaching use of Wikipedia and other associated factors. Source: Own elaboration.

\begin{tabular}{lccc}
\hline Factors & $\mathbf{r}$ & $\mathbf{n}$ & $\mathbf{p}$-value \\
\hline Quality perception & 0.40 & 874 & 0.000 \\
Perception by colleagues & 0.41 & 872 & 0.000 \\
Use by colleagues & 0.41 & 838 & 0.000 \\
Improving students learning & 0.47 & 882 & 0.000 \\
Editing Wikipedia & 0.59 & 887 & 0.000 \\
Consulting Wikipedia for personal matters & 0.30 & 886 & 0.000 \\
Feeling comfortable about students using it for their tasks & 0.51 & 878 & 0.000 \\
\hline
\end{tabular}

Another not so predictable relationship has been found between teaching uses and both colleagues' perception and their behavior of use. Those faculty who use Wikipedia in teaching have a more positive view of their colleagues' opinion on Wikipedia $(r=0.41, N=872)$ and also think they use it more frequently $(r=0.41, N=838)$. Noticeably, a moderate correlation is also detected between the assessment of quality and the perception of colleagues' opinion $(r=0.42, N=874)$ and use $(r=0.37$, $N=840$ ). Those who attribute higher quality to Wikipedia also think their colleagues share a positive view and make regular use of it.

This association, together with the findings presented in section 4.6, supports the idea that academic colleagues are a strong role model for faculty members. Not only does their eventual decision to use Wikipedia as a teaching tool seem to be substantially related to their perception of what colleagues do and think, but also their perception of Wikipedia's overall quality is also linked to their opinion about what colleagues believe.

Finally, other expected associations have also been found with the teaching use of Wikipedia - though correlations are moderate. First, with the opinion that using Wikipedia improves students learning processes $(r=0.47, N=882)$. Second, with seeing no problem when students use it as a source of information $(r=0.51, N=878)$.

\subsection{Teaching use of Wikipedia and faculty features}

We have also investigated to what extent certain faculty personal and professional characteristics are associated with the latent variable measuring teaching uses of Wikipedia. This has been done by estimating an analysis of variance (ANOVA) for testing statistical significance of differences in the mean value among some categories of individuals. In particular we have explored the following categories: gender, academic discipline, years of teaching experience and age. We can only detect significant differences among our respondents $(N=869)$ in the case of gender $(F=17.50, p<0.001)$ and academic discipline $(F=7.53, p<0.01)$.

First of all we should mention that the list of academic disciplines provided in our survey was designed taking into account the main schools of both universities in the survey. That said, the analysis shows that faculty members belonging to STEM fields (in our case natural sciences and engineering disciplines) are the ones with a highest score in teaching practices involving Wikipedia (mean 4.379, $N=206$ ); for other disciplines (in our case mainly humanities, social sciences and law) the mean value was $3.804(N=685)$. This is consistent with former studies - particularly Eijkman (2010) - that have found a more sceptical or negative view on Wikipedia among "soft sciences" faculty than among their "hard sciences" colleagues.

As expected, gender differences were also found e.g. men being more prone to develop teaching uses of Wikipedia - we should remember that a vast majority of Wikipedia editors are known to be male (Antin et al., 2011). Nevertheless, since the population of women in engineering and natural sciences 
is low ( $16.7 \%$ in our sample, while $42.5 \%$ in non-STEM fields), when corrected by the discipline, the gender divide becomes negligible as far as teaching uses of Wikipedia are considered.

Finally, since the influence of age and teaching experience was not found to be significant we can state that faculty's decision to use Wikipedia in learning processes does not follow the usual pattern of other Web 2.0 tools where young people tend to be more frequent users.

\section{Discussion}

There are a number of general conclusions to be drawn from the analysis presented above. First of all, as we expected, few faculty members use Wikipedia for teaching purposes. From those who actually do, most restrict themselves to passive use, that is, basically consulting Wikipedia for elaborating teaching materials. An even smaller minority use it as a platform for developing students' assignments that may involve editing, improving or contributing content. We have found, however, that most faculty show a positive view on the teaching usefulness of Wikipedia; so there is a sharp contrast between their a priori cognitive disposition and their eventual engagement in actual teaching practices.

Contrary to widespread opinion, the results of our survey do not support an overwhelming negative or skeptical attitude among university faculty towards Wikipedia. On the one hand, not only do most of them see it as a useful teaching recourse but few feel uncomfortable about students using it as a source of information. On the other hand, and most surprisingly, data on quality assessment and on use practices plainly contradict the commonly accepted negative standpoint.

The overall quality of Wikipedia articles is rather positively valued. From the three questions asking about quality issues, reliability and updating got a striking majority of positive answers, and only articles' comprehensiveness received a slightly negative evaluation. The common assumption that most faculty members perceive Wikipedia as an inaccurate and unreliable source of information is not supported by our survey's results. Therefore, a negative assessment of quality cannot be taken as the main reason to explain low teaching use rates.

When considering their level of use of Wikipedia both for professional and personal matters but without focusing specifically on teaching activities, faculty members show a similar behaviour to that reported from empirical studies on university students. Most of them are regular users of Wikipedia. In fact, our control data even show that a relatively high percentage of them (13.5\%) are even registered users of Wikipedia - far beyond the average rate of registered users for the general population of Catalonia: $0.4 \%$. All in all, our survey depicts faculty members as frequent users of Wikipedia as long as passive use - consulting or browsing - is concerned.

Nevertheless, we have detected signs of a certain conflict between standard academic procedures of knowledge building and the open collaborative model of peer production on which Wikipedia rests. This is consistent with some of the previous literature we have discussed and it is supported by different findings in our survey. First, their overall assessment of quality of Wikipedia content is much higher than their appreciation of the editing and reviewing system of Wikipedia, which is regarded with distrust. They are more concerned about how articles are built and published than about their quality. Since only very few faculty is actually involved in contributing to Wikipedia - either in improving existing content or in providing new one - this distrust in editing mechanisms is probably not built on accurate knowledge. In fact, in the interviews we conducted before launching the survey, we found several wrong assumptions about the working of Wikipedia, particularly on the editing procedures and on the mechanisms for quality control.

Second, private instances of use - whether professional or personal - are not matched by public uses - those uses that required some sort of publicly stated commitment. Most faculty are definitely not prone to accept students quoting or citing Wikipedia in their assignments, nor do they see it as legitimate to cite or make references to Wikipedia in their own research or teaching. While frequently using it in the private sphere, most faculty think Wikipedia is not well regarded by their colleagues as a respectable source of information. Although they find it useful and rich, they do not tend to recommend its use to students and even less to their colleagues. They are frequent users but prefer not to talk about it. In general, this deep dependence on colleagues' opinion and behaviour, together with the preference for private uses of Wikipedia - those not involving public acknowledgement -, create a negative feedback loop. Since colleagues do not talk much about it, most faculty tend to think their 
colleagues do not use it because they find it inappropriate or unreliable and that prevents them to publicly expose their own positive opinion and intensive use.

In fact, colleagues seem to act as strong role models for most faculty members on this issue, whereas the institutional context - their own university policies and culture - seems less important. Even quality assessment of Wikipedia content seems to be dependent on their perception of colleagues' position about it. Consistent with that, belonging to a specific area of expertise seems to be more decisive than formal institutional affiliation. Like some of the past literature, our study finds that faculty from hard sciences show a more positive assessment and use of Wikipedia than their soft sciences colleagues. The general clash between scientific culture and peer production we had hypothesized is eventually modulated by the particular subcultures of more specific scientific disciplines. As former studies on science culture have demonstrated (Knorr-Cetina, 1992), there are enormous disparities - in methods, practices and epistemological styles - between different sciences.

A final remark can be made on the benefits of actively using Wikipedia in higher education. Though previous studies have successfully highlighted the positive educational improvement of different students' abilities, such as motivation, collaboration, critical reviewing, writing and referencing skills (An and Williams, 2010, Saorín Pérez et al. 2011, Konieczny 2014), much less has been said on the urgency for scientists and scholars to pay attention to Wikipedia as a new and powerful channel for the public communication of science. The widespread social use of Wikipedia as a source for scientific information - including sensitive information on medical and health issues - for the general public, should also encourage a more active and systematic engagement of professional scientists and scholars in improving this free encyclopaedia's content in science-related matters.

\section{Notes}

[1] When we just mention agreement or disagreement, we consider values 4 and 5 for "agree" and values 1 and 2 for "disagree". The remaining percentage corresponds to the middle option (value 3 ).

[2] We omit $p$ value for the rest of correlations since it is always $p<0.001$.

\section{References}

Alonso, M. I. and García, J. (2013), "Colaboración activa en Wikipedia como método de aprendizaje", Revista Iberoamericana de Educación a Distancia, Vol. 16 No. 1, pp. 13-26.

An, Y. and Williams, K. (2010), "Teaching with Web 2.0 Technologies: Benefits, Barriers and Lessons Learned", International Journal of Instructional Technology \& Distance Learning, Vol. 7 No. 3, pp. 4158.

Antin, J. et al. (2011), "Gender differences in Wikipedia editing", in Proceedings of the 7th International Symposium on Wikis and Open Collaboration, Mountain View, CA, ACM, New York, pp. 11-14.

Bayliss, G. (2013), "Exploring the Cautionary Attitude toward Wikipedia in Higher Education: Implications for Higher Education Institutions", New Review of Academic Librarianship, Vol. 19 No. 1, pp. 36-57.

Benkler, Y. (2006), The Wealth of Networks: How Social Production Transforms Markets and Freedom, Yale University Press, New Haven, CT.

Black, E.W. (2008), "Wikipedia and Academic Peer-Review - Wikipedia as a Recognized Medium for Scholarly Publication?", Online Information Review, Vol. 32 No. 1, pp. 73-88. 
Brossard, D. and Scheufele, D.A. (2013), "Science, new media, and the public", Science, Vol. 339 No. 6115, pp. 40-41.

Brox, H. (2012), "The Elephant in the Room: a Place for Wikipedia in Higher Education?", Nordlit, No. 30, pp. 143-155.

Chen, H. (2010), "The Perspectives of Higher Education Faculty on Wikipedia", The Electronic Library, Vol. 28 No. 3, pp. 361-373.

Chen, S. (2010), "Wikipedia: a Republic of Science Democratized", Albany Law Journal of Science and Technology, Vol. 20, No. 2, pp. 249-326.

Dooley, P. (2010), "Wikipedia and the two-faced professoriate", in Wikisym'10 proceedings of the $16^{\text {th }}$ International Symposium on Wikis and Open Collaboration in Gdansk, Poland 2007, Article No. 24, ACM New York, NY, available at: http://dl.acm.org/citation.cfm?id=1832803 (accessed 13 December 2013).

Eijkman, H. (2010), "Academics and Wikipedia: Reframing Web 2.0+ as a Disruptor of Traditional Academic Power-Knowledge Arrangements", Campus-Wide Information Systems, Vol. 27 No. 3, pp. 173-185.

Jaschick, S. (2007), "A stand against Wikipedia", Inside Higher Education, No. 26, available at: http://www.insidehighered.com/news/2007/01/26/wiki (accessed 13 December 2013).

Knight, C. and Pryke, S. (2012), "Wikipedia and the University, a case study", Teaching in Higher Education, Vol. 17 No. 6, pp. 649-659.

Knorr-Cetina, K., (1992), "The Couch, the Cathedral, and the Laboratory: On the Relationship between Experiment and Laboratory in Science", in Pickering, A. (Ed.), Science as practice and culture, The University of Chicago Press, Chicago, IL, pp.113-138.

Konieczny, P. (2014), "Rethinking Wikipedia for the Classroom", Contexts, Vol. 13 No. 1, pp. 80-83.

Lim, S. (2009), "How and Why Do College Students Use Wikipedia?", Journal of the American Society for Information Science and Technology, Vol. 60 No. 11, pp. 2189-2222.

Nielsen, M. (2011), Reinventing Discovery: the New Era of Networked Science, Princeton University Press, Princeton (NJ).

Saorín Pérez, T., de Haro, M.V. and Pastor Sánchez, J.A. (2011), "Posibilidades de Wikipedia en la docencia universitaria: elaboración colaborativa de conocimiento", Ibersid, Vol. 5, pp. 89-97.

Wannemacher, K. and Schulenburg, F. (2010), "Wikipedia in Academic Studies: Corrupting or Improving the Quality of Teaching and Learning?", in Ebner, M. and Schiefner, M. (Eds.), Looking Toward the Future of Technology-Enhanced Education: Ubiquitous Learning and the Digital Native, Information Science Reference, Hershey, PA, pp. 295-311.

Weber, S. (2004), The Success of Open Source, Harvard U.P., Cambridge, MA. 